\title{
ON THE DEGREE OF THE BRANDT-LICKORISH-MILLETT-HO POLYNOMIAL OF A LINK
}

\author{
MARK E. KIDWELL
}

\begin{abstract}
Let $Q_{L}$ be the link polynomial defined by Brandt, Lickorish, Millett, and Ho. Let $\operatorname{deg} Q_{L}$ be the maximum degree of a nonzero term. If $p(L)$ is any regular link projection and $B$ is any bridge (maximal connected component after undercrossing points are deleted), define the length of $B$ as the number of crossings in which the overcrossing segment is a part of $B$.

THEOREM 1. Let $p(L)$ be a connected, regular link projection with $N$ crossing points. Let $K$ be the maximal length of any bridge in $p(L)$. Then $\operatorname{deg} Q_{L} \leq N-K$.

THEOREM 2. If $p(L)$ is a prime, connected alternating projection with $N>0$ crossing points, then the coefficient of $x^{N-1}$ is a positive number.
\end{abstract}

The Laurent polynomial $Q$ of Brandt, Lickorish and Millett [BLM], and Ho $[\mathbf{H}]$ was one of several link invariants to be discovered during the explosion of results following Jones' important work of 1984 . The polynomial is defined inductively by $Q_{U}(x)=1$, where $U$ is the the unknot, and by the relation

$$
Q_{L_{+}}(x)+Q_{L_{-}}(x)=x\left(Q_{L_{0}}(x)+Q_{L_{\infty}}(x)\right),
$$

where $L_{+}$and $L_{-}$are related by a crossing switch at one "site" and $L_{0}$ and $L_{\infty}$ are the two possible eliminations of that crossing (see Figure 1). String orientations and the orientation of 3-space play no role in this definition. The proof of the existence and invariance of $Q$ (see $[\mathbf{B L M}]$ ) is similar to that for other link polynomials obeying relations analogous to (1).

In the planar projections with respect to which $L_{+}, L_{-}, L_{0}$, and $L_{\infty}$ are defined, $L_{+}$and $L_{-}$have one more crossing point than $L_{0}$ and $L_{\infty}$. Since the polynomials $Q_{L_{0}}$ and $Q_{L_{\infty}}$ are multiplied by $x$ in (1), it is intuitively plausible that the degree of the $Q$-polynomial counts the number of crossings in these projections. Of course this cannot always be true because the $Q$-polynomial is an ambient isotopy invariant and the crossing number of a projection is not. We must hope instead that the degree of $Q$ counts the minimal crossing number, a venerable invariant which until recently has been computed only for relatively simple link types in an ad hoc manner.

In actuality, the degree of the $Q$-polynomial gives a lower bound on the difference between the crossing number and another number associated to link projections. We decompose a projection into bridges and define the length of a bridge as the number of crossings in which the bridge forms the overcrossing segment. We then prove that the degree of the $Q$-polynomial is less than or equal to $N-K$, where $N$ is the crossing number and $K$ is the length of the longest bridge of the projection.

Received by the editors April 16, 1986.

1980 Mathematics Subject Classification (1985 Revision). Primary 57M25.

Key words and phrases. Brandt-Lickorish-Millett-Ho polynomial, alternating link projection, bridge length, arborescent link. 

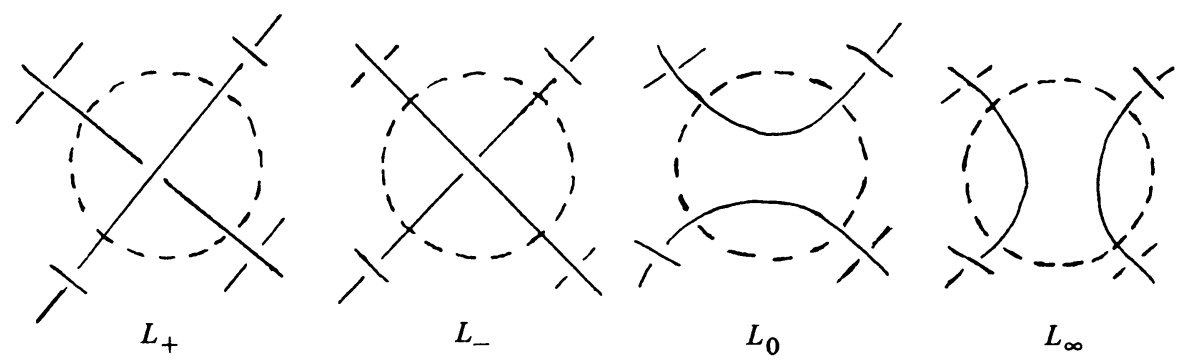

FIGURE 1

An alternating link projection may be defined as one in which all bridges have length 1 . We go on to prove that if $p(L)$ is a prime, connected alternating projection with $N$ crossings, then the degree of the $Q$-polynomial is exactly $N-1$. (The definition of a prime projection is given in $\S 1$.)

Murasugi [M], Kauffman [K], and Thistlethwaite $[\mathbf{T}]$ have recently derived similar results for the Jones polynomial, but without reference to bridge length. They derive a number of geometric consequences which follow also from our results. For example, it follows that a prime alternating link has an alternating projection with fewer crossing points than any nonalternating projection of the same link.

Rather than duplicate these results, we investigate what our results have to say about the Conway notation for a link $[\mathbf{C}]$. If $L$ is a prime, unsplittable alternating link type, the leading coefficient of $Q_{L}$ will turn out to be positive and, if $L$ is nontrivial, even. One can eliminate two-sided regions (clasps) from a suitable projection of such a link without changing this leading coefficient. Since an algebraic tangle $[\mathbf{C}]$ with more than one crossing always contains a clasp, the leading coeffiecient of the $Q$-polynomial depends only on the underlying "basic polyhedron" of the link. In particular, this coefficient is 2 for nontrivial arborescent links.

The author would like to thank Kenneth Millett for suggesting the problem solved in this paper and Bruce Richter and Craig Bailey for useful discussions.

1. Definitions and preliminary results. In this paper, a knot will be regarded as a special kind of link. The number $\operatorname{deg} Q$ will refer to the highest power of $x$ that appears in $Q(x)$, even if $Q$ has terms of negative degree. (It is known that for a link of $l$ components, the lowest power of $x$ that appears in $Q$ is $1-l$.)

We shall work throughout in the piecewise linear category. If $p$ is a regular planar projection of a link $L$, then $N(p(L))$ (or just $N$ if the context is clear) will stand for the number of crossings in the projection.

If we delete the undercrossing points of $L$ with respect to a regular projection $p$, we partition the rest of $L$ into open segments and simple closed curves. (The simple closed curves occur only when $p(L)$ contains an unknotted component in standard position which lies above or is disjoint from all other components.) The projections of the elements of this partition are called the bridges of $p(L)$. The length of a bridge $B$, denoted $k(B)$, is the number of crossings in which the overcrossing segment is a part of $B$. (Traditionally, in defining the bridge number of a projection, bridges of length zero are not counted. We have no reason to exclude them.)

We shall call a bridge $B$ improper if:

(a) $B$ is a simple closed curve and $k(B)>0$; or 


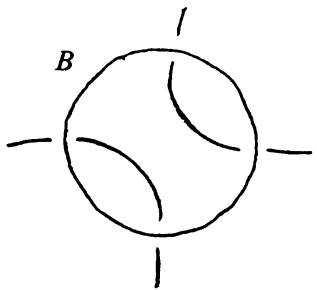

(a)

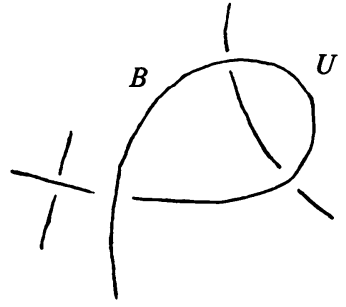

(b)

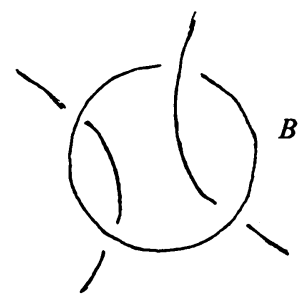

(c)

FIGURE 2

(b) $B$ is an open segment and one or both of the crossings at which $B$ terminates also has $B$ over-crossing; or

(c) the crossings at which $B$ terminates are identical and $k(B)>1$ (see Figure $2(\mathrm{a})-(\mathrm{c}))$.

LEMMA 0. If $p(L)$ contains an improper bridge $B$, then there is a projection $p^{\prime}$ of $L$ containing a bridge $B^{\prime}$ such that $N\left(p^{\prime}(L)\right)<N(p(L))$ and $N\left(p^{\prime}(L)\right)-k\left(B^{\prime}\right) \leq$ $N(p(L))-k(B)$.

PROOF. In case (a), let $c$ be any noncrossing point of $B$. By a combination of Reidemeister moves II and III, $B$ can be contracted to a small crossing-free circle $B^{\prime}$ near $c$. Thus $k\left(B^{\prime}\right)=0$ and $N\left(p^{\prime}(L)\right)=N(p(L))-k(B)$. In case (b) let $c$ be a crossing at which $B$ terminates and overcrosses and let $U$ be a loop of $B$ with no undercrossings from $c$ to $c$. By a combination of Reidemeister moves II and III, $U$ can be contracted to small loop whose only crossing point is $c$. If this sequence of moves reduces $N(P(L))$ and $k(B)$, it reduces them equally. If not, then Reidemeister move I can be applied to the loop $U$. $B$ is replaced by a bridge $B^{\prime}$ with $k\left(B^{\prime}\right) \leq k(B)-1$. Thus

$$
N\left(p^{\prime}(L)\right)-k\left(B^{\prime}\right) \leq(N(p(L))-1)-(k(B)-1)=N(p(L))-k(B) .
$$

In case (c), let $c$ be the crossing at which $B$ terminates. By a combination of Reidemeister moves II and III, $B$ can be contracted to a bridge $B^{\prime}$ of length 1 . $N(p(L))$ and $k(B)$ are reduced equally.

The following trichotomy differs slightly from traditional definitions, but seems most useful for our purposes. A projection $p(L)$ is crossing free if $N(p(L))=0$, alternating if $k(B)=1$ for all bridges $B$ in $p(L)$, and nonalternating if $k(B)>1$ for at least one bridge. Clearly, a crossing-free projection represents a trivial link type. A link type possessing an alternating projection will be called alternating.

We shall have little need to consider the $Q$-polynomials of disconnected link types, since the formula $Q\left(L_{1} \amalg L_{2}\right)=Q\left(L_{1}\right) \mu Q\left(L_{2}\right)$ of [BLM], where $\mu=2 x^{-1}-$ 1 , tells us that the degree of $Q\left(L_{1} \amalg L_{2}\right)$ is the sum of the degrees of $Q\left(L_{1}\right)$ and $Q\left(L_{2}\right)$.

A projection $p(L)$ will be called composite if there is a simple closed curve $C$ in the plane of the projection which intersects $p(L)$ transversely in two points, and both int $C$ and ext $C$ contain crossings of $p(L)$. A projection which is not composite 


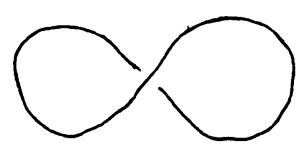

FIGURE 3

is called prime. Note that a prime link type can have a composite projection if one of the "factors" of $p(L)$ is a nontrivial projection of a trivial arc. In particular, any projection with a trivial loop is composite, with the lone exception of the "lemniscate" projection of the unknot (Figure 3). This projection, although it does not display minimal crossing number, is a prime, alternating, connected projection whose $Q$-polynomial has degree $N-1$. Moreover, it is the only connected projection with crossing number 1 . Hence it can be used at the base of some of our inductions.

The paper [BLM] also provides a formula for the $Q$-polynomial of a link with composite projection, namely $Q\left(L_{1} \# L_{2}\right)=Q\left(L_{1}\right) Q\left(L_{2}\right)$. Note that if $\operatorname{deg} Q\left(L_{1}\right)=$ $N\left(p\left(L_{1}\right)\right)-1$ and $\operatorname{deg} Q\left(L_{2}\right)=N\left(p\left(L_{2}\right)\right)-1$, then

$$
\operatorname{deg} Q\left(L_{1} \# L_{2}\right)=N\left(p\left(L_{1}\right)\right)+N\left(p\left(L_{2}\right)\right)-2
$$

while

$$
N\left(p\left(L_{1} \# L_{2}\right)\right)=N\left(p\left(L_{1}\right)\right)+N\left(p\left(L_{2}\right)\right)
$$

Thus the restriction to prime projections in our Theorem 2 is necessary. In general, the $Q$-polynomial is unable to distinguish alternating composite links from nonalternating composite links, as the granny knot and square knot show.

\section{The main results.}

THEOREM 1. Let $p(L)$ be a regular link projection with $N$ crossing points. Let $K$ be the maximal length of any bridge in $p(L)$. Then $\operatorname{deg} Q_{L} \leq N-K$.

PROOF. We shall prove that the set of projections which violate the theorem is empty. Among all projections which are counterexamples to the theorem, consider those with the smallest possible number of crossings and, among those, select a projection $p(L)$ with a bridge of the longest possible length $K$. Thus $\operatorname{deg} Q_{L}>$ $N-K$ with $N$ minimal and $K$ maximal.

Let $B$ be a bridge of length $K$ in $p(L)$. If $B$ is improper, then by Lemma 0 there is a projection $p^{\prime}(L)$ with bridge $B^{\prime}$ such that $N\left(p^{\prime}(L)\right)-k\left(B^{\prime}\right) \leq N-K<\operatorname{deg} Q_{L}$ and $N\left(p^{\prime}(L)\right)<N$, contradicting the minimality of $N$. If $K=0$, then $p(L)$ must be the trivial projection of an unlink, $N=0, \operatorname{deg} Q_{L}=0$, and this is not a counterexample to the theorem. We thus have that $B$ is an open segment and if $c$ is one of its terminal crossings, then the overcrossing segment at $c$ is not a part of $B$ (see Figure 4).

If we perform the operations of equation (1) using $c$ as site, then the projections $p\left(L_{0}\right)$ and $p\left(L_{\infty}\right)$ will have bridges of length at least $K$ and crossing number $N-1$. Thus $\operatorname{deg} Q_{L_{0}} \leq N-1-K$ and similarly for $Q_{L_{\infty}}$. Let $a x^{M}$ be a nonzero term of $Q_{L}=Q_{L_{+}}$with $M>N-K$. Then by (1), the $x^{M}$ term of $Q_{L_{-}}$must be $-a x^{M}$, since $Q_{L_{0}}$ and $Q_{L_{\infty}}$, even multiplied by $x$, cannot contribute to a term of degree 


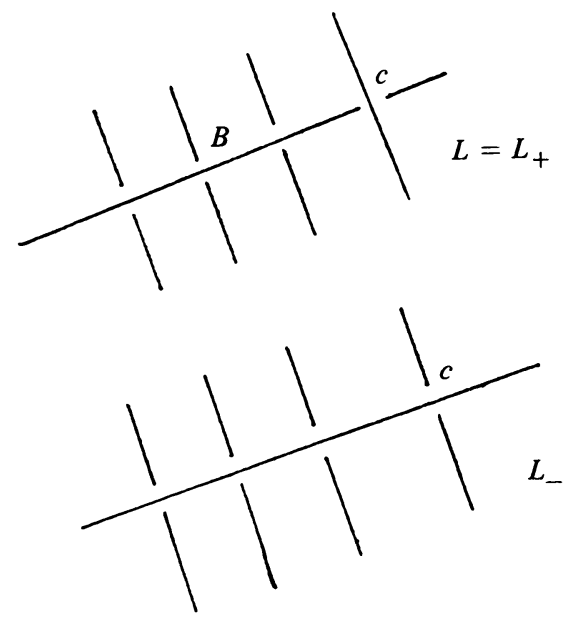

FIGURE 4

$M$. But $p\left(L_{-}\right)$has a bridge of length at least $K+1$, and $\operatorname{deg} Q_{L_{-}} \geq M>N-K>$ $N-(K+1)$. Thus $p\left(L_{-}\right)$is a counterexample to the theorem, contradicting the maximality of $K$.

THEOREM 2. If $p(L)$ is a prime, connected alternating projection with $N$ crossing points, then the coefficient of $x^{N-1}$ in $Q_{L}(x)$ is a positive number.

PROOF. The theorem is true for the "lemniscate" projection of the unknot. Assume the theorem is true for any projection with fewer than $N$ crossings. Take any crossing $c$ in $p(L)=p\left(L_{+}\right)$as site and perform the operatons of (1). Then as Figure 1 indicates, $p\left(L_{-}\right)$has $N$ crossings and a bridge of length 3 , so that $\operatorname{deg} Q_{L_{-}} \leq N-3$. By (1), the coeffiecient of $x^{N-1}$ in $Q_{L}$ will equal the sum of the coefficients of $x^{N-2}$ in $Q_{L_{0}}$ and $Q_{L_{\infty}}$.

Since $p\left(L_{0}\right)$ and $p\left(L_{\infty}\right)$ are alternating projections and have $N-1$ crossings (see Figure 1), our induction hypothesis will complete the proof if we can guarantee that at least one of $p\left(L_{0}\right), p\left(L_{\infty}\right)$ is a prime, connected projection. We prove this combinatorial lemma in an appendix to this paper.

These two theorems apply equally well, and with identical proofs, to the $x$-degree of the Kauffman polynomial $F(a, x)$.

We now investigate how the Brandt-Lickorish-Millett-Ho polynomial relates to the Conway notation for a link. If $p(L)$ is an alternating regular link projection, a region in $R^{2}-p(L)$ having just two crossing points on its boundary is called a clasp. If one applies the operations of (1) to one of the crossings bounding a clasp (see Figure 5), then in one of the two projections eliminating that crossing (we shall call it $\left.p\left(L_{\infty}\right)\right)$, the clasp is replaced with a trivial loop and at least one other crossing can be removed from $p\left(L_{\infty}\right)$ by a type I Reidemeister move. The switched projection $p\left(L_{-}\right)$can be simplified by a type II Reidemeister move.

If the original projection-with-clasp $p\left(L_{+}\right)$is alternating, then the other three projections of formula (1) are, after simplification, alternating with fewer crossings. We shall call the operation of replacing $p\left(L_{+}\right)$with $p\left(L_{0}\right)$ a clasp elimination.

Any link projection having a standard Hopf link factor must be regarded as exceptional in that two clasps share an edge. $p\left(L_{0}\right)$ and $p\left(L_{\infty}\right)$ are not distinguished 


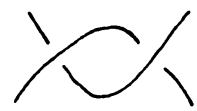

$p\left(L_{+}\right)$
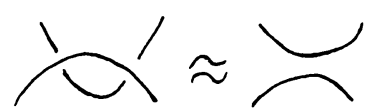

$p\left(L_{-}\right)$

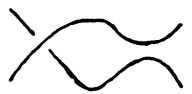

$p\left(L_{0}\right)$

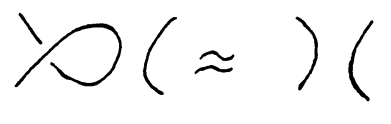

$p\left(L_{\infty}\right)$

\section{FIGURE 5}

unless we specify which clasp we are eliminating. Both projections contain trivial loops.

LEMMA 3. Let $p(L)$ be a prime, connected alternating projection with $N \geq 3$ crossings and a clasp. If $L_{0}$ is any link obtained by eliminating a clasp from $p(L)$, then $Q_{L}$ has the same leading coefficient as $Q_{L_{0}}$.

Proof. By Lemma $\mathrm{A}$ of the appendix, $p\left(L_{0}\right)$ is a prime, connected alternating projection with $N-1$ crossings. By Theorem 2, the leading terms of $Q_{L}$ and $Q_{L_{0}}$ are of the form $a x^{N-1}$ and $b x^{N-2}$ respectively. After the simplifications shown in Figure 5, the maximum nonzero powers of $Q_{L_{\infty}}$ and $Q_{L_{-}}$are at most $x^{N-3}$. By formula (1), $a=b$.

COROLLARY 4. If a prime, connected alternating link projection $p(L)$ can be reduced to the Hopf link by a sequence of clasp eliminations, then the leading coefficient of $Q_{L}$ is 2 .

Proof. The value of the $Q$-polynomial for the Hopf link $2_{1}^{2}$ (see [BLM]) is $-2 x^{-1}+1+2 x$.

COROLLARY 5. If $L$ has an alternating projection $p(L)$ which displays $L$ as a nontrivial arborescent link, then the leading coefficient of $Q_{L}$ is 2 .

ProOF. Any "rational tangle summand" of $L$ can be written in Conway's notation as $M_{1} M_{2} \cdots M_{p}$, with the $M_{i}$ positive integers. Clasp eliminations can reduce this tangle to a string of $p$ 1's. There is still a clasp between the first two crossings in this tangle, so that it can be reduced to 1 or 11 as we choose. Doing the same to any other summand, we arrive at a $(2, q)$ torus link which can be reduced to the Hopf link.

The "algebraic tangles" which close up to arborescent links are the building blocks of what we call a Conway projection of a link. One starts with a 4-valent planar graph with all regions having at least three sides (called a basic polyhedron by Conway), replaces each vertex of the graph by a disk, and embeds an algebraic tangle into each disk so that arcs match.

COROLlaRY 6. If L has a Conway projection which is prime, connected, and alternating, then the leading coefficient of $Q_{L}$ depends only on the basic polyhedron.

PROOF. Each algebraic tangle can be reduced to a single crossing by clasp eliminations, as in Corollary 5. 
In Table 1, we provide a list of the leading coefficients of $Q$-polynomials (for prime alternating links) for the basic polyhedra used by Conway in his enumeration of knots and links. It would be interesting to know whether the nontrivial arborescent links are characterized as those with leading coefficient 2 in their $Q$-polynomials.

TABLE 1

$\begin{array}{cc}\text { Basic Polyhedron } & \text { Leading Coefficient of } Q_{L} \\ 1^{*} & 2 \\ 6^{*} & 4 \\ 8^{*} & 6 \\ 9^{*} & 8 \\ 10^{*} & 8 \\ 10^{* *} & 10 \\ 10^{* * *} & 8 \\ 11^{*} & 12\end{array}$

Appendix. A combinatorial lemma.

LEMMA A. If $p(L)$ is a prime, connected link projection with a crossing $c$ and $p\left(L_{0}\right)$ and $p\left(L_{\infty}\right)$ are the two smoothings of that crossing, then at least on of $p\left(L_{0}\right)$, $p\left(L_{\infty}\right)$ is prime and connected.

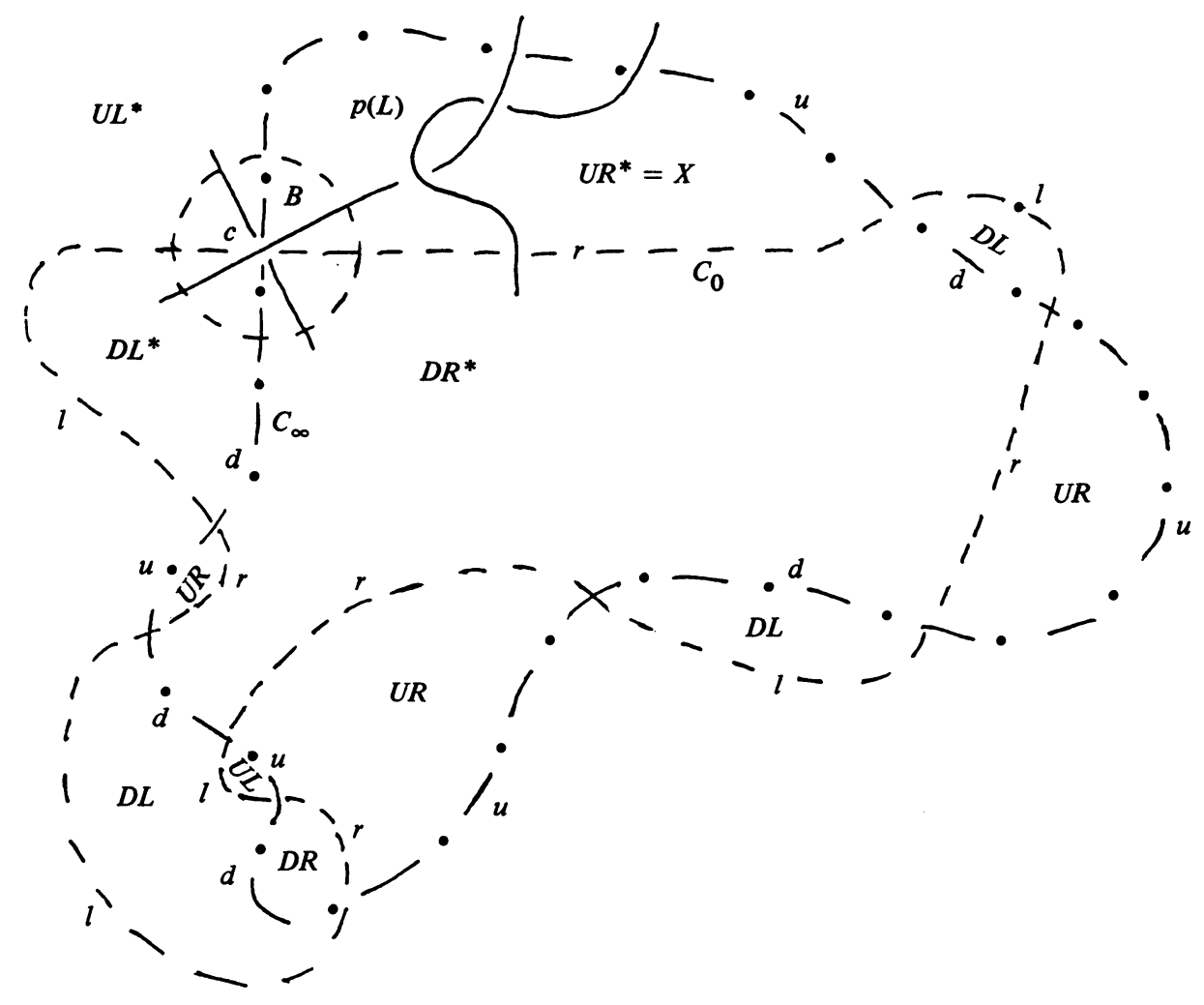

FIGURE 6 
PROOF. If $p\left(L_{0}\right), p\left(L_{\infty}\right)$ were both composite, then there would be separating simple closed curves $C_{0}$ and $C_{\infty}$ in the plane of the projections. Since $p(L)$ is prime, these curves must pass through the site where $p(L)$ differs from $p\left(L_{0}\right)$ and $p\left(L_{\infty}\right)$ as shown in Figure 6. We name four regions (not necessarily connected) in the plane complement of $C_{0} \cup C_{\infty}$ : Int $C_{0} \cap \operatorname{Int} C_{\infty}=\mathrm{DR}$ (for down right), Int $C_{0} \cap \operatorname{Ext} C_{\infty}=$ DL (down left), Ext $C_{0} \cap \operatorname{Int} C_{\infty}=\mathrm{UR}$ (up right), Ext $C_{0} \cap \operatorname{Ext} C_{\infty}=\mathrm{UL}$ (up left).

The connected components of these regions that have crossing $c$ on their boundaries are called $\mathrm{DR}^{* *}, \mathrm{DL}^{* *}, \mathrm{UR}^{* *}$, and $\mathrm{UL}^{* *}$. Let $B$ be a small disk centered at $c$ that only intersects these four regions, and is such that $p(L)$ intersects $\partial B$ in exactly four points. Let $\mathrm{DR}^{*}=\mathrm{DR}^{* *}-B$, etc.

The unions of segments of $C_{0}$ and $C_{\infty}$ are labeled as follows: $\overline{\mathrm{DR}} \cap \overline{\mathrm{DL}}=$ $d, \overline{\mathrm{DR}} \cap \overline{\mathrm{UR}}=r, \overline{\mathrm{DL}} \cap \overline{\mathrm{UL}}=l$, and $\overline{\mathrm{UR}} \cap \overline{\mathrm{UL}}=u$. Note that $C_{0}=r \cup l, C_{\infty}=u \cup d$.

Any connected component $X$ of the complement of $C_{0} \cup C_{\infty} \cup B$ must have an even number of points of $p(L)$ on its boundary. Assume there are crossings of $p(L)$ inside $X$. Then the number of points of $p(L)$ on $\partial X$ must be at least four, or else $\partial X$ would form a separation of $p(L)$, which is assumed prime. ( $X$ cannot contain all crossings of $P(L)$, since it does not contain $c$.) Assume for convenience that $X \subset \mathrm{UR}$, so that $\partial X \subset u \cup r$. At most one of the segments of $p(L) \cap B$ that form crossing $c$ intersects $X$. Thus $\partial X$ must contain at least three points of $(u \cup r) \cap p(L)$.

By the definition of connected sum, $C_{0}$ and $C_{\infty}$ each contain two points of $p(L)$ other than $c$. Thus $u$ and $r$ each contain at most two points of $p(L)-\{c\}$. Let us suppose that $\partial X$ contains two points of $u \cap p(L)$ and at least one of $r \cap p(L)$. Then $d \cap p(L)=\varnothing$ and $l \cap p(L)$ contains at most one point other that $c$.

Let $Y$ be any region in Int $C_{0}-B=(\mathrm{DL} \cup \mathrm{DR})-B$ that contains crossings. (There must be one by the definition of connected sum.) Then as above, $\partial Y \cap p(L)$ must contain at least four points. At most one of these points can be in $\partial Y \cap \partial B$ and at most two can be from $l$ or $r$. (The two can occur only if $Y \subset \mathrm{DR}$ and $\partial X$ and $\partial Y$ share an edge.) But since $d \cap p(L)=\varnothing, \partial y \cap p(L)$ cannot have four points.

A similar contradiction arises if any other assumption is made about the intersection of $P(L)$ with $u, d, r$, and $l$ or if $p\left(L_{0}\right)$ or $p\left(L_{\infty}\right)$ is assumed to be disconnected rather than composite.

\section{REFERENCES}

[BLM] R. D. Brandt, W. B. R Lickorish and K. C. Millett, A polynomial invariant for unoriented knots and links, Invent. Math. 84 (1986), 563-573.

[C] J. H. Conway, An enumeration of knots and links and some of their algebraic properties, Computational Problems in Abstract Algebra (J. Leech, ed.), Pergamon Press, Oxford and Elmsford, N.Y., 1969, pp. 329-358.

[H] C. F. Ho, A new polynomial invariant for knots and links, Abstracts Amer. Math. Soc. 6 (1985), 300 .

[K] L. Kauffman, State models and the Jones polynomial (to appear).

[M] K. Murasugi, Jones polynomials and classical conjectures in knot theory, Topology (to appear).

[T] M. B. Thistlethwaite, A spanning tree expansion of the Jones polynomial, Topology (to appear).

Department of Mathematics, United States Naval Academy, Annapolis, MARYLAND 21402 